with special reference to butter fat. Ind. and Eng. Chemistry, juin 1924, 16, 598 .

[5] L. A. Rogers. Factors influencing changes in storage butter. $I I I^{\text {e }}$ Congrès international de la Réfrigération, Washington, septembre-octobre, 1916.

[6] O. F. Hunziker et D. Fay Hosman. Tallowy butter. Its causes and prevention. Jour. of Dairy Sc., novembre 1917, 1, 4.

[7] L. H. Briggs. The autoxidation of butterfat. The Jour. of Dairy Research, décembre 1931, 3, 1, 61-79.

[8] Wright et Overman. Effet du cuivre, de l'acide lactique et de la tempéra ture sur l'auto-oxydation de la graisse de beurre et du saindoux. 1927.

[9] K. E. Wright. A study of some of the factors affecting the autoxidation of butter fat. Thèse, Urbana (Illinois), 1927.

[10] A. H. Solway. Studies on the oxidation of unsaturated fatty oils and unsaturated fatty acids. Jour. of the Chem. Soc., 1923, 109, $1^{\text {re }}$ partie, 138.

[11] Holm et GreEnbank. Quantitative aspects of the Kreist test. Ind. and Eng. Chemistry, octobre 1923, 15, 10, 1051 ; et mai 1924, 16, 5, 518.

[12] Taffel et Revis. La détermination de la rancidité dans les huiles et les graisses. Jour. de la Soc. de Chim. ind., 5087 T, 1931. Ext. des Matières Grasses, 15 mai 1931, 277 ; et 15 juillet 1931, 279.

\title{
UNE NOUVELLE MÉTHODE DE DOSAGE DES PROTIDES DU LAIT
}

\author{
par Lascar Buruiana.
}

(Laboratoire de Chimie Biologique, Faculté de Médecine Vétérinaire de Bucarest.)

Historique. - L'action de l'acide nitrique sur les protides a fait l'objet de nombreuses recherches. Dès 1799, Hatchetr s'intéresse à l'étude des composés nitro-protéiques (cité par HaBERManN et EHRENFELD).

L'acide nitrique a d'ailleurs une action spécifique sur les protides. Si l'on fait bouillir ces substances additionnées de cet acide, elles prennent une coloration jaune claire qui passe, par alcalinisation à la soude, au jaune foncé. Cette réaction, connue sous le nom de xantho-protéique, fait partie des réactions colorées des protides.

Selon le but envisagé, les études faites sur l'action de l'acide nitrique sur les protides peuvent se diviser en :

$1^{0}$ Etudes concernant la séparation des composés qui résultent de cette action variable (xanthoprotéines, acides xanthoprotéiques, nitro-albumoses, nitroprotéoses $[1,2]$ etc.). Ces composés varient avee les conditions du travail, la concentration de l'acide, le temps d'action, le mode de séparation et la nature des matières premières. Une importance spéciale pour notre étude revient à l'action de l'acide nitrique concentré. Cette action peut se diviser en : $a$ ) hydro[ysante ; $b$ ) de nitration ; $c$ ) oxydante. 
Cette dernière action peut être réduite au minimum, comme nous avons pu le constater au cours de ce travail. Elle est due, moins à l'acide nitrique lui-même qu'aux oxydes d'azote qui proviennent de sa décomposition.

L'identification des composés résultant de la nitration a fait l'objet de nombreuses et minutieuses recherches entreprises par C. Th. Mörner et ses collaborateurs. Ainsi, on a pu distinguer : l'acide picrique, le p. nitrobenzène, l'acide benzoïque, l'acide téréphtalique, l'acide oxalique, etc., etc.

$2^{\circ}$ Etudes concernant l'explication chimique de la coloration produite dans la réaction xanto-protéique.

INOUYE [3] attribua, le premier, la coloration orange à la nitration des acides oxyaromatiques qui résultent de l'hydrolyse des protides. D'après lui, le grand responsable est la tyrosine. Plus tard, RoDhe, SALKowsky, ABDERHALDEN et Kemp attribuèrent aussi au tryptophane la production de cette coloration. C. Th. Mörner, dans un travail ( Welchen Anteil haben Tyrosin und Tryptophan an den Farben Effekt bei den beiden Phasen der Xanthoproteisaurereaktion "), a établi que, en ce qui concerne la contribution de ces amino-acides à l'intensité de la coloration dans les deux phases (acide et alcaline), existait le rapport suivant: Tyrosine acide/Tryptophane acide = $1 / 3$ et tryptophane ammoniacal/tyrosine ammoniacale $=9 / 45$. On voit que le virage est plus accentué pour la tyrosine que pour le tryptophane.

On comprend aisément que, en dehors de ces composés, il en puisse exister une foule d'autres qui contribuent à la réaction xantho-protéique. L'identification des nitro-dérivés résultants est très difficile, à cause de la diversité des composés qui peuvent apparaître à la suite de la nitration, et aussi, parce que la nitration des amino-acides n'a pas encore fait l'objet d'un travail systématique et complet.

OBJET DE CE TRAVAIL. - Dans le présent travail, nous avons cherché à établir, à l'aide de cette réaction colorée, une méthode en vue du dosage des substances protéiques du lait. Presque toutes les méthodes actuelles de dosage se ramènent à la précipitation des protides du lait par des agents physico-chimiques et à la détermination, soit du poids du précipité obtenu, soit de l'azote contenu dans ce précipité, à l'aide de la méthode de KJELDAHL. Notons des variantes volumétriques de ces procédés parmi lesquelles il faut citer spécialement la méthode de DENIG Ès. Les réactions colorées des substances protéiques ont été très peu utilisées pour leur dosage [5]. Parmi elles, la réaction xantho-protéique nous a paru convenir le mieux parce qu'elle réunissait toutes les conditions de rapidité et de simplicité. Il restait à établir que cette réaction pouvait servir 
pour le dosage colorimétrique, c'est-à-dire qu'il existe une proportionnalité réelle entre l'intensité de la coloration et la quantité de substances protéiques utilisée. Dans ce but, nous avons préparé une solution titrée de caséine très pure, dont la teneur en azote avait été établie par la méthode de KJеLdahl. Par une série d'expériences, nous avons déterminé :

Io La concentration optimum de l'acide nitrique. Nous avons choisi, au début, l'acide concentré qui assure une parfaite solubilisation des substances protéiques et une nitration complète et uniforme. Nous ne craignons pas de répéter ici que l'action oxydante de l'acide nitrique sur les protéines est due en premier lieu aux produits de décomposition de cet acide, c'est-à-dire aux oxydes d'azote. Si l'on fait bouillir, par exemple, les substances protéiques additionnées d'acide nitrique dans un ballon KJELDAHL, l'espace disponible qui se trouve au-dessus du liquide favorise la formation des vapeurs nitreuses.

Après refroidissement et neutralisation à la soude, on n'obtient pas une belle coloration orange comme d'habitude, mais une couleur brun-rougeâtre, caractéristique pour les composés résultant d'une oxydation déjà avancée. Si l'on fait bouillir, au contraire, dans une éprouvette fermée par un bouchon en caoutchouc muni d'un réfrigérant à air de $1 \mathrm{~m}$. de longueur, la décomposition est presque nulle, et l'action oxydante très réduite, ce qui permet d'obtenir, dans tous les cas, la même couleur orange, très belle.

$2^{\circ}$ Le temps d'ébullition. Les expériences faites en ce qui concerne le temps d'ébullition ont donné des résultats identiques si l'on fait bouillir pendant 1, 2, 3 ou 4 minutes. Le chauffage prolongé provoque une diminution de l'intensité de la coloration, due à l'oxydation qui commence à se faire sentir. Nous avons choisi finalement, comme temps d'ébullition, deux minutes.

$3^{\circ}$ La quantité d'acide. La quantité d'acide a peu d'importance. En général, une quantité de $3 \mathrm{cmc}$. d'acide nitrique pour $30 \mathrm{mgr}$. de caséine constitue un minimum. Nous avons employé, dans tous les cas, des quantités d'acide variant entre 5 et $7 \mathrm{cmc}$. pour $30 \mathrm{mgr}$. de caséine.

$4^{\circ}$ La réaction du milieu. L'intensité de la coloration étant plus grande dans la deuxième phase de la réaction, il faut alcaliniser après ébullition et refroidissement, avant de faire les comparaisons colorimétriques.

La potasse caustique est à rejeter parce qu'elle provoque une mousse abondante pendant la neutralisation, et dont le débordement hors du ballon est à craindre.

L'ammoniaque n'est pas à recommander, parce que la couleur 
obtenue après neutralisation est faible, en comparaison avec celle obtenue après neutralisation par les hydrates alcalins.

Dans tous les cas, nous avons employé la soude caustique concentrée (à $40 \%$ ).

Technique du Dosage. -- Tenant compte des études préliminaires ainsi faites, nous avons établi la technique suivante :

On mesure, avec une pipette très exacte, $1 \mathrm{cmc}$. de la solution de caséine, dans une éprouvette de verre d'IENa de dimensions $200 \times 23 \mathrm{~mm}$.

On ajoute 5 à $6 \mathrm{cmc}$, d'acide azotique concentré et l'on ferme l'éprouvette avec un bouchon en caoutchouc muni d'un réfrigérant ascendant à air de 1 mètre de longueur. Ayant fixé le tout, incliné à. un support, on fait bouillir pendant deux minutes, exactement. On refroidit au robinet et l'on transvase le liquide résultant dans un matras jaugé, de 50 cmc.

On neutralise dans ce ballon même, avec la soude à $40 \%$, toujours en refroidissant. L'opération est terminée quand la couleur du contenu du ballon passe du jaune pâle à l'orange intense. Le virage s'observe parfaitement. On filtre rapidement sur amiante. Le liquide obtenu doit être parfaitement limpide.

Résultats. Pour vérifier s'il y a proportionnalité entre la coloration obtenue et la quantité de caséine prise, nous avons répété l'opération avec des quantités différentes. Les résultats obtenus sont consignés dans le tableau suivant:

Tableau I.

\begin{tabular}{r|c|c|c|c|r|r}
\hline Cme. sol. de caséine à 3\% $\%$ & 0,25 & 0,50 & 1 & 1,50 & 2 & 3 \\
Cme. NO ${ }^{3} \mathrm{H}$ (densité : 1,39).. & 3 & 3 & 5 & 7,5 & 10 & 15 \\
Porté en matras jaugé de : c.. & 25 & 50 & 50 & 50 & 100 & 150 \\
Intensité en mm. au colori- & & & & & & \\
mètre Dubosq ........ & 7,5 & 7,5 & 15 & 10 & 15 & 15 \\
\hline \hline
\end{tabular}

On voit, d'après ce tableau, que les résultats sont très satisfaisants, la proportionnalité étant strictement réalisée. Pour les déterminations colorimétriques, nous avons recouru à un étalon fixe et facile à préparer. Après de nombreuses recherches, nous avons adopté le mélange utilisé déjà par C. Th. MöRner. Cet étalon, qui contient : 31,0 cme. d'une solution decinormale de bichromate de potasse +4 cc. 5 d'une solution centinormale de permanganate de potasse - le tout additionné d'eau distillée jusqu'au volume de 50 cmc., donne la même teinte que celle présentée par $30 \mathrm{mgr}$ de caséine traités comme il a été mentionné plus haut. Cette solution étalon n'est pas parfaitement stable parce que le permanganate de potasse précipite du bioxyde de manganèse sur les parois du ballon. 
Pour éviter cet inconvénient, nous recommandons de préparer l'étalon au moment du dosage.

Avec cette solution type, nous avons comparé les couleurs obtenues avec des quantités variables de caséine. Les résultats sont représentés dans la fig. 1 suivante :

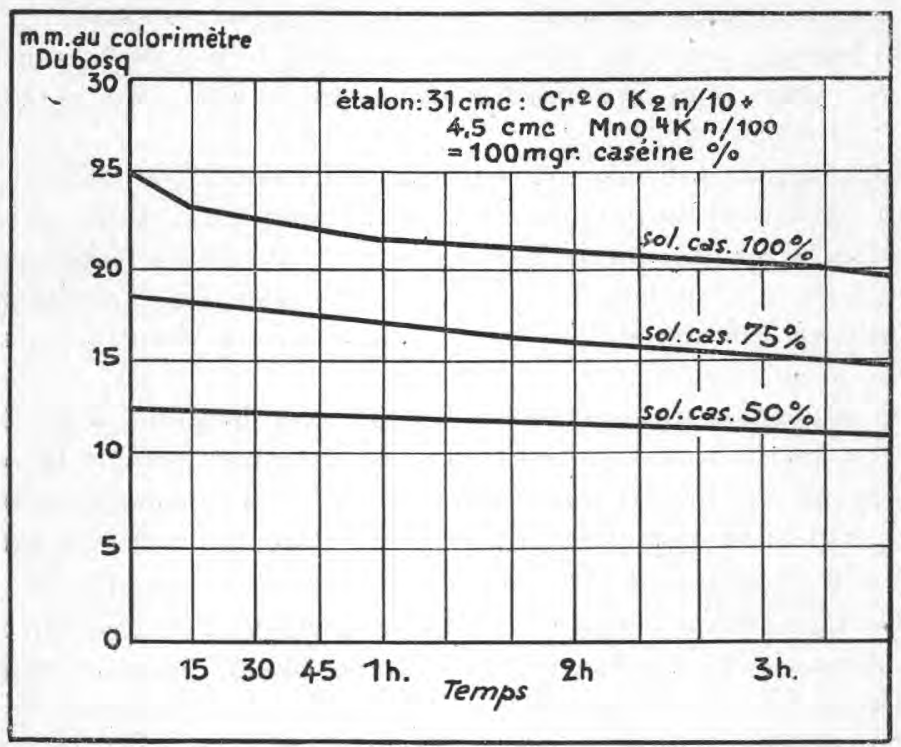

En étudiant les courbes de la figure 1, on constate que :

10 La couleur n'est pas stable;

$2^{\circ}$ La couleur s'atténue avec le temps, le maximum d'intensité ayant lieu immédiatement après la neutralisation ;

$3^{\circ}$ La vitesse de décoloration est proportionnelle à la concentration en substance protéique ;

Les conclusions qu'on peut tirer de ces observations, pour assurer un dosage exact, sont les suivantes :

$1^{\circ}$ Les mesures colorimétriques doivent être faites immédiatement après neutralisation avec la soude. Il ne faut pas attendre plus de quinze minutes.

$2^{\circ}$ La concentration finale (après neutralisation dans le matras jaugé) en matière protéique ne doit pas dépasser $70 \mathrm{mgr}$ pour $100 \mathrm{cc}$.

DOSAGE DES PROTIDES DU LAIT. - Ces résultats satisfaisants nous ont décidé à appliquer cette méthode au dosage des protéines totales du lait et de la caséine. Nous avons pensé que la méthode serait particulièrement intéressante si l'on pouvait l'appliquer 
directement au lait tel qu'il est. Mais, d'abord, il était nécessaire d'étudier l'influence des autres constituants du lait.

10 Dans le lait on trouve des protéines autres que la caséine qui pourraient donner une réaction xantho-protéique différente comme intensité de celle donnée par la caséine. En expérimentant avec ees substances séparément, nous avons constaté une très petite différence en ce qui concerne l'intensité de la réaction xantho-protéique. L'erreur faite en considérant toutes les protéines comme caséine est. donc très petite, surtout dans le cas où la quantité des protéines autres que la caséine est petite par rapport à cette dernière.

On peut d'ailleurs établir l'étalon en dosant les protéines du lait par la méthode KJELDAHL.

$2^{\circ}$ Les graisses et les liquides, en général, n’interviennent pas du tout dans cette réaction.

$3^{\circ}$ Le lactose peut, lors de la neutralisation, donner une coloration jaune brunâtre qui peut modifier celle produite par la réaction xantho-protéique si l'on n'a pas soin de bien refroidir le liquide pendant la neutralisation avec de la soude. La température ne doit pas dépasser $30^{\circ} \mathrm{C}$, sinon les résultats sont faussés. Les autres constituants du lait n'interviennent pas dans la réaction xantho-protéique.

En nous basant sur ces études préliminaires, nous avons établi la technique suivante pour le dosage des protéines du lait:

On introduit dans une éprouvette de verre d'IENA de dimensions $200 \times 23 \mathrm{~mm}$., $1 \mathrm{cmc}$. de lait, exactement mesuré, et 6 à $7 \mathrm{cmc}$. d'acide nitrique concentré de densité 1,39-1.40, puis l'on procède comme dans le cas des solutions de caséine.

On fait bouillir pendant 2 minutes exactement, on refroldit, et l'on transvase le liquide dans un matras jaugé de 50 ou de $100 \mathrm{cmc}$. (la concentration en matière protéique ne doit pas dépasser $70 \mathrm{mgr} \%$ ). On neutralise dans le ballon même avec une solution de soude concentrée à $40 \%$, en ajoutant un léger excès. On complète jusqu'au trait, et, après agitation, on filtre sur amiante. La vitesse de filtration doit être de 40 à $50 \mathrm{cmc}$. en 5 minutes.

Le liquide, qui doit être limpide, est porté rapidement dans la cuve du colorimètre et comparé ¿̀ la solution étalon $(18,6 \mathrm{cc}$. de solution décinormale de bichromate de potasse $+2,7 \mathrm{cc}$. de solution centinormale de permenganate de potasse) - le tout complété, avec de l'eau distillée, à $100 \mathrm{cc}$. La coloration de cette solution étalon est la même que celle présentée par $60 \mathrm{mgr}$ de caséine traitée comme il est dit plus haut dans un ballon jaugé de $100 \mathrm{cc}$.

A l'aide de cette méthode, nous avons fait une série de déterminations. Les résultats sont indiqués dans le tableau II suivant: 
Tableau II.

\begin{tabular}{|c|c|c|c|c|c|}
\hline Cme lait $\ldots \ldots \ldots \ldots \ldots \ldots \ldots$ & 0,25 & 0,50 & 1 & 2 & 4 \\
\hline Cmc. acide nitrique conc. . ...... & 3 & 3 & 6 & 12 & 24 \\
\hline Capacité du ballon jaugé, en ec..... & 25 & 50 & 100 & 100 & 250 \\
\hline Gr. caséine au litre $\ldots \ldots \ldots \ldots \ldots$ & 33,2 & 33,50 & 33,50 & 33,1 & 33,2 \\
\hline
\end{tabular}

Nous avons appliqué cette méthode au dosage des protéines du lait et des caséines. Les résultats ont été contrôlés simultanément par ceux donnés par les méthodes Denigìs et KJeldahl. Dans le tableau III, nous donnons les résultats de quelques déterminations :

Tableau III.

\begin{tabular}{|c|c|c|c|c|}
\hline No & & Méth. Kjeldahl & Méth. Denigès & Méth. à $\mathrm{NO}_{3} \mathrm{H}$ conc. \\
\hline 1 & Lait de vache ... & 一 & 28,0 gr. $\%$ & 28,50 gr. $\%$ \\
\hline 2 & $\ldots$ & 一 & 30,75 & 31 \\
\hline 3 & $\cdots$ & 一 & 32 & 32 \\
\hline 4 & $\ldots$ & 一 & 40 & 41 \\
\hline 5 & Lait de bufflesse.. & 一 & 38 & 39 \\
\hline 6 & $\ldots$ & - & 40,50 & 41 \\
\hline 7 & $\ldots$ & - & 42 & 42 \\
\hline 8 & $\ldots$ & - & 45 & 46 \\
\hline 9 & $\ldots$ & - & 35 & 41 \\
\hline 10 & $\cdots$ & 一 & 34 & 41 \\
\hline 11 & $\begin{array}{r}\text { Caséine Hammars- } \\
\text { ten .......... }\end{array}$ & - & 88 & 88,50 \\
\hline 12 & Caséine industrielle & 一 & 80,50 & 7985 \\
\hline
\end{tabular}

Les résultats obtenus avec la nouvelle méthode sont aussi précis que ceux obtenus avec les autres méthodes. Nous attirons l'attention sur les cas 9 et 10 du tableau précédent. On observe, dans ces cas, une différence frappante. En cherchant les causes de cette différence, nous avons pu constater les choses suivantes : le lait analysé avait été préalablement gelé (les déterminations étant faites pendant l'hiver). Ce changement accidentel pourrait être la cause de perturbations dans la structure physique des colloïdes protéiques, entraînant un comportement différent lors de la précipitation ultérieure par le réactif iodo-mercurique (c'est ce réactif que l'on utilise dans la méthode de Denigìs).

Dosage de la caséine Seule. - On peut doser la caséine du lait en procédant comme suit : 
On dilue $1 \mathrm{cmc}$. de lait dans $4 \mathrm{cmc}$. d'eau distillée et l'on ajoute jusqu'à précipitation totale $(\mathrm{pH} 4,6)$ de l'acide acétique à $2 \%$ (5 à 6 gouttes).

On filtre, sur un filtre plissé de $6 \mathrm{cmc}$. de diamètre, et l'on rince, avec quelques cmc. d'eau chaude, les particules du précipité restées sur les parois de l'éprouvette dans laquelle a eu lieu la précipitation. Après écoulement complet du filtrat, on introduit le filtre avec le précipité dans l'éprouvette de dosage. On ajoute 5 à 6 cmc. d'acide azotique concentré et l'on continue l'opération comme il est dit plus haut.

La différence obtenue entre les protéines totales et la caséine seule nous révèle les protéines autres que la caséine.

\section{CONCLUSIONS.}

Dans ce qui précède, nous avons décrit une nouvelle méthode colorimétrique pour le dosage des protides du lait. La méthode est basée sur la réaction xantho-protéique. Elle a le grand avantage de la simplicité, de la rapidité et de l'exactitude. Pour obtenir toujours des résultats satisfaisants, il faut respecter les conditions suivantes :

$1^{\circ}$ La concentration en substances protéiques prises dans l'analyse ne doit pas dépasser $70 \mathrm{mgr}$ pour $100 \mathrm{ec}$.

$2^{\circ}$ La neutralisation et l'alcalinisation à la soude doivent se faire en ayant soin de bien refroidir. Autrement, et spécialement dans le cas du lait total, la présence du lactose peut fausser les résultats.

$3^{\circ}$ Les mesures colorimétriques doivent être faites le plus rapidement possible, tout au plus 15 minutes après la neutralisation, la couleur s'atténuant avec le temps.

\section{BIBLIOGRAPHIE.}

[1] FürTH. Habilitationsschrift, Strasbourg, 1899.

[2] Limben. Biochem. Zeitsch., pp. 535-559, 1924.

[3] Inouye. Hoppe Seyler Zeitsch. f. physiol. Chemie, 35, 308, 1902 et 45, p. 579, 1905.

[4] C. Th. Mörner. Zeitsch. f. physiol, Chemie, 107, 203, 1919.

[ō] Autenrieth. Munch. med. Wsch, 62e année, Cahier 42, 1417 (1915) et $64^{\mathrm{e}}$ année, Cahier 8, 241 (1917).

[6] Huller. Proceed. of the Soc. f. exper. Biol. a. Med. 24, 385 (1927). 